\title{
THE INVERSE MEAN CURVATURE FLOW IN ROBERTSON-WALKER SPACES AND ITS APPLICATION TO COSMOLOGY*
}

\author{
CLAUS GERHARDT ${ }^{\dagger}$
}

\begin{abstract}
We consider the inverse mean curvature flow in Robertson-Walker spacetimes that satisfy the Einstein equations and have a big crunch singularity and prove that under natural conditions the rescaled inverse mean curvature flow provides a smooth transition from big crunch to big bang. We also construct an example showing that in general the transition flow is only of class $C^{3}$.
\end{abstract}

Key words. Lorentzian manifold, transition from big crunch to big bang, cyclic universe, general relativity, inverse mean curvature flow, ARW spacetimes

AMS subject classifications. 35J60, 53C21, 53C44, 53C50, 58J05

0. Introduction. In a series of papers we considered the problem of finding a smooth transition from a spacetime with a big crunch to a spacetime with a big bang in a classical setting assuming that the Einstein equations and the equation of state for a perfect fluid are valid in a (punctured) neighbourhood of the singularity. We distinguished two cases, depending if the spacetime could be embedded as a hypersurface or brane in a bulk spacetime, or if it was merely given as an abstract globally hyperbolic Lorentzian manifold.

In the case of branes embedded in the black hole region of an $\mathrm{AdS}_{n+2}$ bulk spacetime $\mathcal{N}$, which is topologically a product $\mathcal{N}=\mathbb{R}_{-} \times \mathbb{R} \times \mathcal{S}_{0}$, where $\mathcal{S}_{0}$ is an $n$ dimensional space form, we could show that, assuming the usual isotropy and spherical symmetry of the branes, the brane could be extended smoothly past the singularity into a new bulk space $\hat{\mathcal{N}}=\mathbb{R}_{+} \times \mathbb{R} \times \mathcal{S}_{0}$. The new extended hypersurface was smoothly embedded into

$$
\mathcal{N} \cup \hat{\mathcal{N}}=\mathbb{R}^{2} \times \mathcal{S}_{0}
$$

only the induced metric had a singularity corresponding to the black (white) hole singularity of the bulk spacetimes, for details cf. [4] and especially [5].

For abstract spacetimes with a big crunch singularity, that are not realized as hypersurfaces in a bulk spacetime, it is already a non-trivial matter how to define a smooth transition past the singularity.

In a recent paper [2] we proved that in this situation the inverse mean curvature flow, properly rescaled, could be used to define a smooth transition from a big crunch spacetime to a big bang spacetime, and we also showed that the transition flow was of class $C^{3}$. The underlying $(n+1)$-dimensional spacetime $N$ was fairly general, a cosmological spacetime satisfying the structural conditions stated below, we called these spacetimes ARW spaces.

Definition 0.1. A globally hyperbolic spacetime $N, \operatorname{dim} N=n+1$, is said to be asymptotically Robertson-Walker (ARW) with respect to the future, if a future end of $N, N_{+}$, can be written as a product $N_{+}=[a, b) \times \mathcal{S}_{0}$, where $\mathcal{S}_{0}$ is a Riemannian

\footnotetext{
*Received August 6, 2004; accepted for publication March 23, 2006.

${ }^{\dagger}$ Ruprecht-Karls-Universität, Institut für Angewandte Mathematik, Im Neuenheimer Feld 294, 69120 Heidelberg, Germany (gerhardt@math.uni-heidelberg.de). This work has been supported by the Deutsche Forschungsgemeinschaft.
} 
space, and there exists a future directed time function $\tau=x^{0}$ such that the metric in $N_{+}$can be written as

$$
d \breve{s}^{2}=e^{2 \tilde{\psi}}\left\{-\left(d x^{0}\right)^{2}+\sigma_{i j}\left(x^{0}, x\right) d x^{i} d x^{j}\right\},
$$

where $\mathcal{S}_{0}$ corresponds to $x^{0}=a, \tilde{\psi}$ is of the form

$$
\tilde{\psi}\left(x^{0}, x\right)=f\left(x^{0}\right)+\psi\left(x^{0}, x\right),
$$

and we assume that there exists a positive constant $c_{0}$ and a smooth Riemannian metric $\bar{\sigma}_{i j}$ on $\mathcal{S}_{0}$ such that

$$
\lim _{\tau \rightarrow b} e^{\psi}=c_{0} \quad \wedge \quad \lim _{\tau \rightarrow b} \sigma_{i j}(\tau, x)=\bar{\sigma}_{i j}(x),
$$

and

$$
\lim _{\tau \rightarrow b} f(\tau)=-\infty
$$

Without loss of generality we shall assume $c_{0}=1$. Then $N$ is ARW with respect to the future, if the metric is close to the Robertson-Walker metric

$$
d \bar{s}^{2}=e^{2 f}\left\{-d x^{0^{2}}+\bar{\sigma}_{i j}(x) d x^{i} d x^{j}\right\}
$$

near the singularity $\tau=b$. By close we mean that the derivatives of arbitrary order with respect to space and time of the conformal metric $e^{-2 f} \breve{g}_{\alpha \beta}$ in (0.2) should converge to the corresponding derivatives of the conformal limit metric in (0.6), when $x^{0}$ tends to $b$. We emphasize that in our terminology Robertson-Walker metric does not necessarily imply that $\left(\bar{\sigma}_{i j}\right)$ is a metric of constant curvature, it is only the spatial metric of a warped product.

We assume, furthermore, that $f$ satisfies the following five conditions

$$
-f^{\prime}>0 \text {, }
$$

there exists $\tilde{\omega} \in \mathbb{R}$ such that

$$
n+\tilde{\omega}-2>0 \wedge \lim _{\tau \rightarrow b}\left|f^{\prime}\right|^{2} e^{(n+\tilde{\omega}-2) f}=\tilde{m}>0 .
$$

Set $\tilde{\gamma}=\frac{1}{2}(n+\tilde{\omega}-2)$, then there exists the limit

$$
\lim _{\tau \rightarrow b}\left(f^{\prime \prime}+\tilde{\gamma}\left|f^{\prime}\right|^{2}\right)
$$

and

$$
\left|D_{\tau}^{m}\left(f^{\prime \prime}+\tilde{\gamma}\left|f^{\prime}\right|^{2}\right)\right| \leq c_{m}\left|f^{\prime}\right|^{m} \quad \forall m \geq 1,
$$

as well as

$$
\left|D_{\tau}^{m} f\right| \leq c_{m}\left|f^{\prime}\right|^{m} \quad \forall m \geq 1 .
$$

If $\mathcal{S}_{0}$ is compact, then we call $N$ a normalized ARW spacetime, if

$$
\int_{\mathcal{S}_{0}} \sqrt{\operatorname{det} \bar{\sigma}_{i j}}=\left|S^{n}\right| .
$$


In this paper we shall show that in general the differentiability class $C^{3}$ is the best posible for the transition flow. If it should be of class $C^{\infty}$, then additional assumptions have to be satisfied.

We shall consider the problem in Robertson-Walker spaces $N=I \times \mathcal{S}_{0}$, where $\mathcal{S}_{0}$ is a space form with curvature $\tilde{\kappa}=-1,0,1$, it may be compact or not, and the metric in $N$ is of the form

$$
d \bar{s}^{2}=e^{2 f}\left(-\left(d x^{0}\right)^{2}+\sigma_{i j}(x) d x^{i} d x^{j}\right)
$$

where $x^{0}=\tau$ is the time function, $\left(\sigma_{i j}\right)$ the metric of $\mathcal{S}_{0}, f=f(\tau)$, and $x^{0}$ ranges between $-a<x^{0}<0$. We assume that there is a big crunch singularity in $\left\{x^{0}=0\right\}$, i.e., we assume

$$
\lim _{\tau \rightarrow 0} f(\tau)=-\infty \quad \text { and } \quad \lim _{\tau \rightarrow 0}-f^{\prime}=\infty
$$

The Einstein equations should be valid with a cosmological constant $\Lambda$

$$
G_{\alpha \beta}+\Lambda \bar{g}_{\alpha \beta}=\kappa T_{\alpha \beta}, \quad \kappa>0
$$

or equivalently,

$$
G_{\alpha \beta}=\kappa\left(T_{\alpha \beta}-\sigma \bar{g}_{\alpha \beta}\right), \quad \sigma=\frac{\Lambda}{\kappa} .
$$

If $\left(T_{\alpha \beta}\right)$ is the stress-energy tensor of a perfect fluid

$$
T_{0}^{0}=-\rho, \quad T_{i}^{\alpha}=p \delta_{i}^{\alpha}
$$

with an equation of state

$$
p=\frac{\omega}{n} \rho,
$$

then the equation (0.16) is equivalent to the Friedmann equation

$$
\left|f^{\prime}\right|^{2}=-\tilde{\kappa}+\frac{2 \kappa}{n(n-1)}(\rho+\sigma) e^{2 f},
$$

which can be easily derived by looking at the component $\alpha=\beta=0$ in (0.16).

Assuming that $\omega$ is of the form

$$
\omega=\omega_{0}+\lambda(f), \quad \omega_{0}=\mathrm{const},
$$

where $\lambda=\lambda(t)$ is smooth satisfying

$$
\lim _{t \rightarrow-\infty} \lambda(t)=0,
$$

such that there exists a primitive $\tilde{\mu}=\tilde{\mu}(t), \tilde{\mu}^{\prime}=\lambda$, with

$$
\lim _{t \rightarrow-\infty} \tilde{\mu}(t)=0,
$$

then $\rho$ obeys the conservation law

$$
\rho=\rho_{0} e^{-\left(n+\omega_{0}\right) f} e^{-\tilde{\mu}},
$$


cf. [2, Lemma 0.2]. Hence we deduce from (0.19)

$$
\left|f^{\prime}\right|^{2}=-\tilde{\kappa}+\frac{2 \kappa}{n(n-1)}\left(\rho_{0} e^{-\left(n+\omega_{0}\right) f} e^{-\tilde{\mu}}+\sigma\right) e^{2 f} .
$$

The main result of the paper can be summarized in the following theorem

Theorem 0.2. Let $\tilde{\gamma}=\frac{1}{2}\left(n+\omega_{0}-2\right)>0$, and assume that $\lambda$ satisfies the condition (1.2) and that $\mu$ can be viewed as a smooth and even function in the variable $(-r)^{\tilde{\gamma}}$, where $r=-e^{f}<0$, or that it can be extended to a smooth and even function on $\left(-\tilde{\gamma}^{-1}, \tilde{\gamma}^{-1}\right)$, then the transition flow $y=y(s, \xi)$, as defined in (2.16) and (2.17), is smooth in $\left(-\tilde{\gamma}^{-1}, \tilde{\gamma}^{-1}\right) \times \mathcal{S}_{0}$, if either

$$
\omega_{0} \in \mathbb{R} \text { and } \sigma=0,
$$

or

$$
\omega_{0}=4-n \text { and } \quad \sigma \in \mathbb{R} .
$$

Let us emphasize that the smooth transition from big crunch to big bang does not constitute the existence of a cyclic universe, cf. the end of Section 2 for a detailed discussion.

In Section 3 we prove that in general the transition flow is only of class $C^{3}$ by constructing a counter example.

We believe that the results and even more the proofs indicate strongly that the inverse mean curvature flow is the right vehicle to offer a smooth transition from big crunch to big bang in case of abstract spacetimes that are not embedded in a bulk spacetime.

We refer to [1, Section 2] for a description of our notations and conventions.

1. The Friedmann equation. We want to solve the Friedmann equation (0.24) in an interval $I=(-a, 0)$ such that the resulting spacetime $N$ is an ARW space, cf. Definition 0.1.

In a slightly different setting we proved in [2, Section 9] that a cosmological spacetime satisfying the Einstein equations for a perfect fluid with an equation of state (0.18), $\omega=$ const, is an ARW space, if

$$
\tilde{\gamma}=\frac{1}{2}(n+\omega-2)>0 .
$$

This result will also be valid in the present situation.

Lemma 1.1. Let $\tilde{\gamma}=\frac{1}{2}\left(n+\omega_{0}-2\right)$ be positive and assume that $\lambda$ and $\tilde{\mu}$ satisfy the conditions stated in the previous section, and in addition suppose

$$
\left|D^{m} \lambda(t)\right| \leq c_{m} \quad \forall m \in \mathbb{N} .
$$

Then the Friedmann equation (0.24) can be solved in an interval $I=(-a, 0)$ such that $f \in C^{\infty}(I)$ and the relations (0.14) are valid. Moreover, $N$ is an $A R W$ space.

Proof. We want to apply the existence result [4, Theorem 3.1]. Multiply equation $(0.24)$ by $e^{2 \tilde{\gamma} f}$ and set

$$
\varphi=e^{\tilde{\gamma} f}
$$


and

$$
r=-e^{f}
$$

Then $\varphi$ satisfies the differential equation

$$
\tilde{\gamma}^{-2} \dot{\varphi}^{2}=-\tilde{\kappa} e^{2 \tilde{\gamma} f}+\frac{2 \kappa}{n(n-1)}\left(\sigma e^{2(\tilde{\gamma}+1) f}+\rho_{0} e^{-\mu}\right),
$$

where we defined $\mu=\mu(r)$ by

$$
\mu(r)=\tilde{\mu}(\log (-r))
$$

Suppose the Friedmann equation were solvable with $f$ satisfying $(0.14)$, then the right-hand side of (1.5) would tend to $\frac{2 \kappa}{n(n-1)} \rho_{0}$, if $\tau \rightarrow 0$. Thus, we see that solving $(0.24)$ and $(0.14)$ is equivalent to solving

$$
\tilde{\gamma}^{-1} \dot{\varphi}=-\sqrt{F(\varphi)}
$$

with initial value $\varphi(0)=0$, where

$$
F(\varphi)=-\tilde{\kappa} \varphi^{2}+\frac{2 \kappa}{n(n-1)}\left(\rho_{0} e^{-\mu}+\sigma \varphi^{2\left(1+\tilde{\gamma}^{-1}\right)}\right)
$$

and $\mu$ should be considered to depend on

$$
\mu(r)=\mu\left(-\varphi^{\tilde{\gamma}^{-1}}\right) .
$$

We can now apply the existence result in [4, Theorem 3.1] to conclude that $(1.7)$ has a solution $\varphi \in C^{1}((-a, 0]) \cap C^{\infty}((-a, 0))$, where, if we choose $a$ maximal, $a$ is determined by the requirement

$$
\lim _{\tau \rightarrow-a} \varphi=\infty \quad \text { or } \quad \lim _{\tau \rightarrow-a} F(\varphi)=0 .
$$

Set $f=\tilde{\gamma}^{-1} \log \varphi$, then $f$ satisfies $(0.14)$, since $F(0)>0$.

Moreover, differentiating (1.5) with respect to $\tau$ and dividing the resulting equation by $2 f^{\prime} e^{\tilde{\gamma} f}$ we obtain

$$
f^{\prime \prime}+\tilde{\gamma}\left|f^{\prime}\right|^{2}=-\tilde{\kappa} \tilde{\gamma}+\frac{\kappa}{n(n-1)}\left(2 \sigma(\tilde{\gamma}+1) e^{2 f}-\lambda \rho_{0} e^{-\mu}\right)
$$

from which we conclude that $N$ is an ARW space, in view of (0.21), (0.22) and (1.2).

2. The transition flow. Let $M_{0}$ be a spacelike hypersurface with positive mean curvature with respect to the past directed normal, then the inverse mean curvature flow with initial hypersurface $M_{0}$ is given by the evolution equation

$$
\dot{x}=-H^{-1} \nu
$$

where $\nu$ is the past directed normal of the flow hypersurfaces $M(t)$ which are locally defined by an embedding

$$
x=x(t, \xi), \quad \xi=\left(\xi^{i}\right),
$$

cf. [2] for details. 
In general, even in Robertson-Walker spaces, this evolution problem can only be solved, if $\mathcal{S}_{0}$ is compact. However, if, in the present situation, we assume that $M_{0}$ is a coordinate slice $\left\{x^{0}=\right.$ const $\}$, then the fairly complex parabolic system (2.1) is reduced to a scalar ordinary differential equation.

Look at the component $\alpha=0$ in (2.1). Writing the hypersurfaces $M(t)$ as graphs over $\mathcal{S}_{0}$

$$
M(t)=\left\{(u, x): x \in \mathcal{S}_{0}\right\},
$$

we see that $u$ only depends on $t, u=u(t)$, and $u$ satisfies the differential equation

$$
\dot{u}=\frac{1}{-n f^{\prime}},
$$

where $f=f(u)$, with initial value $u(0)=u_{0}$, cf. [2, Section 2]. The mean curvature of the slices $M(t)$ is given by

$$
H=e^{-f}\left(-n f^{\prime}\right) .
$$

From (2.4) we immediately deduce

$$
\frac{d}{d t}(n f+t)=n f^{\prime} \dot{u}+1=0
$$

and hence

$$
e^{n f} e^{t}=\mathrm{const}=c
$$

or equivalently,

$$
e^{\tilde{\gamma} f} e^{\gamma t}=c,
$$

where $\gamma=\frac{1}{n} \tilde{\gamma}$, and where the symbol $c$ may represent different constants.

The conservation law (2.8) can be viewed as the integrated version of the inverse mean curvature flow.

In $\left[2\right.$, Theorem 3.6] we proved that there are positive constants $c_{1}, c_{2}$ such that

$$
-c_{1} \leq \tilde{u} \leq-c_{2}<0 .
$$

The old proof also works in the present situation, where $\mathcal{S}_{0}$ is not necessarily compact, since $u$ doesn't depend on $x$.

Moreover,

$$
\lim _{t \rightarrow \infty} \tilde{u} \text { exists, }
$$

cf. [2, Lemma 7.1].

We shall define a new spacetime $\hat{N}$ by reflection and time reversal such that the IMCF in the old spacetime transforms to an IMCF in the new one.

By switching the light cone we obtain a new spacetime $\hat{N}$. The flow equation in $N$ is independent of the time orientation, and we can write it as

$$
\dot{x}=-H^{-1} \nu=-(-H)^{-1}(-\nu) \equiv-\hat{H}^{-1} \hat{\nu},
$$

where the normal vector $\hat{\nu}=-\nu$ is past directed in $\hat{N}$ and the mean curvature $\hat{H}=-H$ negative. 
Introducing a new time function $\hat{x}^{0}=-x^{0}$ and formally new coordinates $\left(\hat{x}^{\alpha}\right)$ by setting

$$
\hat{x}^{0}=-x^{0}, \quad \hat{x}^{i}=x^{i},
$$

we define a spacetime $\hat{N}$ having the same metric as $N$-only expressed in the new coordinate system - such that the flow equation has the form

$$
\dot{\hat{x}}=-\hat{H}^{-1} \hat{\nu}
$$

where $M(t)=\operatorname{graph} \hat{u}(t), \hat{u}=-u$.

The singularity in $\hat{x}^{0}=0$ is now a past singularity, and can be referred to as a big bang singularity.

The union $N \cup \hat{N}$ is a smooth manifold, topologically a product $(-a, a) \times \mathcal{S}_{0}$-we are well aware that formally the singularity $\{0\} \times \mathcal{S}_{0}$ is not part of the union; equipped with the respective metrics and time orientation it is a spacetime which has a (metric) singularity in $x^{0}=0$. The time function

$$
\hat{x}^{0}=\left\{\begin{aligned}
x^{0}, & \text { in } N, \\
-x^{0}, & \text { in } \hat{N},
\end{aligned}\right.
$$

is smooth across the singularity and future directed.

Using the time function in (2.14) the inverse mean curvature flows in $N$ and $\hat{N}$ can be uniformly expressed in the form

$$
\dot{\hat{x}}=-\hat{H}^{-1} \hat{\nu}
$$

where (2.15) represents the original flow in $N$, if $\hat{x}^{0}<0$, and the flow in (2.13), if $\hat{x}^{0}>0$.

In [2] we then introduced a new flow parameter

$$
s=\left\{\begin{aligned}
-\gamma^{-1} e^{-\gamma t}, & \text { for the flow in } N \\
\gamma^{-1} e^{-\gamma t}, & \text { for the flow in } \hat{N},
\end{aligned}\right.
$$

and defined the flow $y=y(s)$ by $y(s)=\hat{x}(t) . y=y(s)$ is then defined in $\left[-\gamma^{-1}, \gamma^{-1}\right] \times$ $\mathcal{S}_{0}$, smooth in $\{s \neq 0\}$, and satisfies the evolution equation

$$
y^{\prime} \equiv \frac{d}{d s} y=\left\{\begin{aligned}
-\hat{H}^{-1} \hat{\nu} e^{\gamma t}, & s<0 \\
\hat{H}^{-1} \hat{\nu} e^{\gamma t}, & s>0
\end{aligned}\right.
$$

or equivalently, if we only consider the salar version with $\eta=\eta(s)$ representing $y^{0}$

$$
\eta^{\prime}=\frac{d}{d s} \eta=\left\{\begin{aligned}
\dot{u} e^{\gamma t}, & s<0 \\
-\dot{\hat{u}} e^{\gamma t}, & s>0 .
\end{aligned}\right.
$$

According to the results in [2, Theorem 8.1] $y$, and hence $\eta$, are of class $C^{3}$ across the singularity. 
Now, looking at the relation (2.8) we see that the new parameter $s$ could just as well be defined by

$$
s=\left\{\begin{array}{cc}
-\tilde{\gamma}^{-1} e^{\tilde{\gamma} f}, & s<0, \\
\tilde{\gamma}^{-1} e^{\tilde{\gamma} f}, & s>0,
\end{array}\right.
$$

where in $N$ as well as in $\hat{N} f$ is considered to be a function of $u(t), f=f(u(t))$.

Defining $s$ by (2.19) we deduce for $s<0$

$$
\eta^{\prime}=\dot{u} \frac{d t}{d s}=\dot{u} \frac{1}{-f^{\prime} e^{\tilde{\gamma} f} \dot{u}}=\frac{1}{-f^{\prime} e^{\tilde{\gamma} f}} \equiv \varphi^{-1} .
$$

The same relation is also valid for $s>0$.

Suppose now that $\varphi$, or equivalently, $\varphi^{2}$,

$$
\varphi^{2}=\left|f^{\prime}\right|^{2} e^{2 \tilde{\gamma} f}=-\tilde{\kappa} e^{2 \tilde{\gamma} f}+\frac{2 \kappa}{n(n-1)}\left(\sigma e^{2(\tilde{\gamma}+1) f}+\rho_{0} e^{-\mu}\right),
$$

can be viewed as an even function in $e^{\tilde{\gamma} f}$, or equivalently, an even function in $s$, then $\eta$ would be of class $C^{\infty}$ across the singularity, and hence the transition flow $y=y(s)$ would be smooth.

We have thus proved

THEOREM 2.1. Let $\tilde{\gamma}=\frac{1}{2}\left(n+\omega_{0}-2\right)>0$, and assume that $\lambda$ satisfies the condition (1.2) and that $\mu$ is a smooth and even function in the variable $(-r)^{\tilde{\gamma}}, r<0$, or can be extended to a smooth and even function on $\left(-\tilde{\gamma}^{-1}, \tilde{\gamma}^{-1}\right)$, then the transition flow $y=y(s, \xi)$ is smooth in $\left(-\tilde{\gamma}^{-1}, \tilde{\gamma}^{-1}\right) \times \mathcal{S}_{0}$, if either

$$
\omega_{0} \in \mathbb{R} \quad \text { and } \quad \sigma=0,
$$

or

$$
\omega_{0}=4-n \quad \text { and } \quad \sigma \in \mathbb{R} .
$$

If $n=3$ and (2.23) is valid, this means that we consider a radiation dominated universe.

Let us also emphasize that in the preceding theorem we have only proved a smooth transition from big crunch to big bang. This does not necessarily mean that we have a cyclic universe - the same observation also applies to the transition results we obtained in [4] in a brane cosmology setting.

It could well be that the following scenario holds: The spacetime $N$ exists in $-\infty<\tau<0$ with the only singularity in $\tau=0$, a big crunch; the mean curvature of the slices $\left\{x^{0}=\right.$ const $\}$ is always positive and

$$
\lim _{\tau \rightarrow-\infty} e^{f}=\infty
$$

After a smooth transition through the singularity the mirror image $\hat{N}$ develops.

Such a pair of universes $(N, \hat{N})$ can be easily constructed, in fact, this will always be the case, if the right-hand side of equation (1.8) never vanishes and grows at most 
quadratically in $\varphi$, which will be the case, if $\sigma=0$, since then equation (1.7) will be solvable in an interval $(-a, 0]$, where $a$ is determined by the requirement

$$
\lim _{\tau \rightarrow-a} F(\varphi)=0 .
$$

Hence, if $F(\varphi)$ never vanishes, the solution of $(1.7)$ will exist in $(-\infty, 0]$. Moreover, in $\tau=-\infty$ there cannot be a singularity, a big bang, since this would require that the mean curvature of the coordinate slices tend to $-\infty$. But this impossible, since $H$ never changes sign, there exist no maximal hypersurfaces in $N$.

To give an explicit example set $\sigma=\mu=0$ and assume $\tilde{\kappa}=0,-1$. Then equation (1.5) has the form

$$
\dot{\varphi}^{2}=-\tilde{\kappa} \tilde{\gamma}^{2} \varphi^{2}+\frac{2 \kappa \tilde{\gamma}^{2}}{n(n-1)} \rho_{0} .
$$

If $\tilde{\kappa}=-1$, we deduce

$$
\varphi=\lambda \sinh (c \tau), \quad \lambda<0, \quad c>0,
$$

and if $\tilde{\kappa}=0$, then

$$
\dot{\varphi}=-c^{2},
$$

hence

$$
\varphi=-c^{2} \tau
$$

i.e.,

$$
e^{f}=\left(-c^{2} \tau\right)^{\tilde{\gamma}^{-1}}
$$

3. A counter example. We shall show that, even in the case of RobertsonWalker spaces, the transition flow is in general only of class $C^{3}$, by constructing a counter example.

TheOREM 3.1. Let $\omega=\omega_{0}$ be such that

$$
\tilde{\gamma}=\frac{1}{2}(n+\omega-2) \geq 2,
$$

and assume $\sigma \neq 0$. Then the Friedmann equation (0.24) has a solution in the interval $(-a, 0)$ such that corresponding spacetime is an $A R W$ space. The transition flow $y=y(s)$, however, is only of class $C^{3}$. If $\tilde{\gamma}=2$, then $y$ is of class $C^{3,1}$, but, if $\tilde{\gamma}>2$, then

$$
\lim _{s \uparrow 0}\left|\frac{d^{4} \eta}{(d s)^{4}}\right|=\infty,
$$

where $\eta=\eta(s)$ is defined as in (2.18).

Proof. Due to Lemma 1.1 the Friedman equation is solvable and the resulting spacetime is an ARW space.

Notice that

$$
s=\left\{\begin{aligned}
-c e^{\tilde{\gamma} f}, & s<0, \\
c e^{\tilde{\gamma} f}, & s>0,
\end{aligned}\right.
$$


and hence we conclude

$$
\eta^{\prime}(s)=\varphi^{-1}
$$

cf. equation (2.20), where $\varphi^{2}$ can be expressed as

$$
\varphi^{2}=-\tilde{\kappa} c_{1} s^{2}+c_{2} \rho_{0}+c_{3} \sigma\left(s^{2}\right)^{1+\tilde{\gamma}^{-1}}
$$

with positive constants $c_{i}$.

The proof of the theorem can now be completed by elementary calculations. $\square$

\section{REFERENCES}

[1] Claus Gerhardt, Hypersurfaces of prescribed curvature in Lorentzian manifolds, Indiana Univ. Math. J., 49 (2000), pp. 1125-1153, arXiv:math.DG/0409457.

[2] The inverse mean curvature flow in ARW spaces - transition from big crunch to big bang, 2004, arXiv:math.DG/0403485, 39 pages.

[3] _ The inverse mean curvature flow in cosmological spacetimes, 2004, arXiv:math.DG/0403097, 24 pages.

[4] - Transition from big crunch to big bang in brane cosmology, Adv. Theor. Math. Phys., 8 (2004), pp. 319-343, gr-qc/0404061.

[5] Branes, moduli spaces and smooth transition from big crunch to big bang, Adv. Theor. Math. Phys., 10:3 (2006), pp.283-315, arXiv:hep-th/0409123.

[6] Justin Khoury, A briefing on the ekpyrotic/cyclic universe, 2004, 8 pages, astro-ph/0401579.

[7] Neil Turok and Paul J. Steinhardt, Beyond inflation: A cyclic universe scenario, 2004, hep-th/0403020, 27 pages. 